\title{
Inscrutability and the Opacity of Natural Selection and Random Genetic Drift: Distinguishing the Epistemic and Metaphysical Aspects
}

\section{Philippe Huneman}

\section{Erkenntnis}

An International Journal of Scientific

Philosophy

\section{ISSN 0165-0106}

\section{Volume 80}

Supplement 3

Erkenn (2015) 80:491-518

\section{E R K E N N T N I S}

DOI 10.1007/s10670-014-9684-z

AN INTERNATIONAL JOURNAL OF

SCIENTIFIC PHILOSOPHY

Editor-in-chief: Hannes Leitgeb

ON PROBABILITIES IN BIOLOGY AND PHYSICS

Guest Editors:

Joseph Berkovitz

and Philosophy of Science and Technology,

University of Toronto

Philippe Hunema

Institut d'Histoire et de Philosophie des Sciences et des Techniques,

\section{黛 Springer}


Your article is protected by copyright and all rights are held exclusively by Springer Science +Business Media Dordrecht. This e-offprint is for personal use only and shall not be selfarchived in electronic repositories. If you wish to self-archive your article, please use the accepted manuscript version for posting on your own website. You may further deposit the accepted manuscript version in any repository, provided it is only made publicly available 12 months after official publication or later and provided acknowledgement is given to the original source of publication and a link is inserted to the published article on Springer's website. The link must be accompanied by the following text: "The final publication is available at link.springer.com". 


\title{
Inscrutability and the Opacity of Natural Selection and Random Genetic Drift: Distinguishing the Epistemic and Metaphysical Aspects
}

\author{
Philippe Huneman
}

Received: 8 September 2014/ Accepted: 8 September 2014/Published online: 23 September 2014

(C) Springer Science+Business Media Dordrecht 2014

\begin{abstract}
Statisticalists' argue that the individual interactions of organisms taken together constitute natural selection. On this view, natural selection is an aggregated effect of interactions rather than some added cause acting on populations. The statisticalists' view entails that natural selection and drift are indistinguishable aggregated effects of interactions, so that it becomes impossible to make a difference between them. The present paper attempts to make sense of the difference between selection and drift, given the main insights of statisticalism; basically, it will disentangle the various kinds of indistinguishability between selection and drift that happen within biology, by examining the epistemological and metaphysical nature of the distinction between selection and drift. It will be based on a 'difference-making account' of selection. The first section will explicate the inscrutability of selection and drift, its various types in the statisticalist writings, and its implications. The second section specifies concepts of natural selection and drift in the difference making account of selection I am using, and shows that one can derive from this the statistical signatures of selection and drift. On this basis I focus on one sort of indistinguishability issue about selection and drift, which I call epistemic opacity, and explain why it mostly affects small populations. The last section explains why epistemic opacity does not raise an genuine epistemic problem for evolutionary biology.
\end{abstract}

\footnotetext{
P. Huneman $(\bowtie)$

Institut d'Histoire et de Philosophie des Sciences et des Techniques, CNRS/Université Paris I

Sorbonne, Paris, France

e-mail: philippe.huneman@gmail.com
} 


\section{Introduction}

The classical framework of evolutionary theory is a synthesis of Darwinian theory of natural selection and Mendelian theory of genetics. Population genetics in this context is the science of the process of evolution by natural selection. Models in population and quantitative genetics are statistical models, and the main concepts in this theory, such as fitness, are probabilistic. However, the founding fathers of population genetics, notably Wright, Fisher and Haldane, presented the theory in terms of causes and forces: that is, selection, drift and mutation are forces acting on gene pools, causes of alleles or traits frequency changes. The ambiguity between probabilistic and causal terminology remained unnoticed for a long time. Could these different terminologies, which reflect two frameworks of thinking about evolution by natural selection, be reconciled?

In principle, there are two ways of making sense of this ambiguity. The first, somehow inflationist, is to assume that statistical models represent a dynamics in the population of organisms, and that selection, drift and mutation are causes acting in this dynamics; the main version has been elaborated by Sober (1984). The second way is deflationist in that it claims that selection, migration, mutation and drift are not considered as causes but rather statistical relations that are the result of mixing causal processes at the singular level of organisms, and capture them in population genetics' models. Here the whole causal talk is not justified. This statisticalist position has been defended by Lewens, Ariew, Walsh and Matthen and stimulated many responses from the inflationary camp (see e.g. Bouchard and Rosenberg 2004; Stephens 2004; Shapiro and Sober 2007).

In essence, the statisticalists argue that the individual interactions of organisms taken together in aggregate constitute natural selection. Statisticalism makes a strong case for rejecting the idea that selection is some superadded or exogenous force, in addition to the set of interactions within the population of organisms. However, the consequence of this position is that natural selection and drift are indistinguishable aggregated effects of interactions so that it is impossible to make a difference between them.

The focus of this paper is the question: how to make sense of the difference between selection and drift, given the main insights of statisticalism? I will disentangle the various kinds of indistinguishability between natural selection and drift in evolutionary biology by examining the epistemological and metaphysical nature of them. My analysis will rely on a conceptual distinction between selection and drift which is based on a "difference-making account" of selection.

The first section presents the various kinds of the inscrutability of selection and drift according to statisticalism, and their implications for evolutionary biology. Section 3 presents the concepts of natural selection and drift in terms of the difference-making account, and shows how one can derive from them distinct corresponding statistical patterns. Section 4 presents another a kind of inscrutability concerning selection and drift at another level, namely 'epistemic opacity' concerning natural selection, and explains why it mostly affects small populations and can be overcome in many cases, identifying and solving two kinds of problematic cases. 


\section{The Inscrutability of Selection and Drift and the Problem for Philosophy of Biology}

\subsection{The Inscrutability Problem}

Statisticalism about drift and natural selection holds that natural selection is not a separate cause that acts upon populations, but rather a result of the ensemble of individual interactions between organisms ("the trials of life"). "Fitness and natural selection have no reality except as accumulations of more fundamental events" (Matthen and Ariew 2002, 82). Therefore selection and drift are not specific forces, but rather statistical properties of populations. Actually when an ensemble of individuals with heritable variable traits interact and reproduce generation after generation, the average of the expected offspring numbers of the individuals sharing a same trait plays a predictive role. This is what statisticalists called 'trait fitness'; it is thereby not a cause that occurs at the level of individual processes and operate on individual organisms; it is merely a source of predictions about the frequencies of alleles, not a cause of them.

In this perspective, a radical consequence is that natural selection and evolution per se are the same thing. "In this way of looking at things, the distinction between evolution (the total change of gene frequencies due to all causes), and natural selection (the portion of evolution due to differences in competitive advantage) is unmotivated" (Matthen and Ariew 2002, 78) The statisticalists' claim is radical since one of the main tenets of the Modern Synthesis is that "natural selection is not evolution": that was Fisher's claim in the opening sentence of The genetical theory of natural selection, and it was intended to emphasize that understanding how selection works, and how it contributes to evolutionary change or stasis in any given context, is a key task of evolutionary biology. In Fisher's view selection contributes to evolutionary change and biologists should be able to determine how much selection contributes to such change (Fisher also claimed this contribution is always a positive change in mean fitness, as states his famous Fundamental Theorem of Natural Selection).

If the Statisticalists are right, the question of distinguishing selection and drift (that are supposedly two "factors" of evolution) ${ }^{1}$ appears to be meaningless, since natural selection is just evolution. Statisticalists thus subsequently assert at various points that selection and drift are inscrutable. The indistinguishability of selection and drift has three main aspects. I will present them and the rest of the paper will question their validity.

a. The "whether" indistinguishability. First, the indistinguishability between selection and drift can mean that deciding whether it is selection or drift that created an outcome depends upon the way trait fitness is computed, which in turns depend upon the parsing of the environment and the population, so the distinction is just an artefact of the measuring device.

\footnotetext{
1 See Depew (2013) on the terms "factors" and "forces" and their respective historical uses.
} 
The idea is here that whether an outcome is to be attributed to drift or selection is sometimes a matter of explanatory context. (Walsh et al. 2002, 466)

"So the outcome within the patch is either drift or selection depending upon how the trait fitnesses are calculated." (ibid.) ${ }^{2}$

b. The "how much" indistinguishability. Second, in a way Statisticalists indicate is somehow different from the latter question of deciding between selection and drift, there is the assertion that there is no objective (vs. epistemic) apportion of responsibility (so here it is a quantitative issue concerning the evaluation of the role of random genetic drift rather than detecting its presence per se that is at stake).

"No objective, as opposed to epistemic, apportioning of causal responsibility to selection as against drift in a concrete evolutionary history." (Matthen and Ariew 2002, 62)

"The proposition that drift was involved to degree $\mathrm{p}$ in this history generally has no determinate truth value." (Matthen and Ariew 2002, 65)

Statisticalists also strongly suggest this claim is supported by general considerations about probabilities: "It would be a mistake, however, to ask how much chance contributed to the actual history of human hairlessness, just as it would be a mistake to try to apportion the role of chance in a particular series of coin tosses" (Matthen and Ariew 2002, 64) This resonates with Beatty's view that "it is difficult to distinguish between random drift on the one hand, and the improbable results of natural selection on the other hand", since "all the outcomes within the ranges of outcomes of natural selection" are indeed possible outcomes of natural selection (Beatty 1994, 196); statisticalists do not think it is merely "difficult" - rather they argue it is impossible. c. The "target" indistinguishability. Third, indistinguishability can mean that it makes no sense to look for the targets of selection and the targets of drift, since, selection being a probabilistic feature, it can't be responsible of one individual event rather than of another one. "One cannot point to a single event of, say, a birth or death and ask whether it is a consequence of the process of natural selection" (Walsh et al. 2002, 453) ${ }^{3}$ Thus, this inscrutability, as I call it from now, is justified by the nature of probability (what Matthen and Ariew call "sound probabilistic thinking"). If a dice with a bias on 6 rolls, it is wrong to

\footnotetext{
2 Along this line Walsh $(2007,2010)$ further developed a powerful argument against the causal conception of selection, that is addressed in Huneman (2013) along the lines of this paper.

3 See also: "So it may be tempting to say that natural selection is the cause of evolution (...) and that it consists, over a longer period of time, of "predictable" (or fitness- biased) cases (...), but that it excludes anomalous (or fitness-indiscriminate) cases (...). Then it might be thought plausible to say that something else-drift? indiscriminate, or neutral, selection? -is operating in (the latter cases). But this violates sound probabilistic thinking." (Matthen and Ariew 2002, 62) "Although it is six times more probable that two heads will turn up in a run of four tosses of a coin than that four will, chance does not play any more of a role in a particular run of four heads than in a particular run of two and two. Thus, one cannot in general differentiate between individual events on the basis of how much they are attributable to chance." (Matthen and Ariew 2002, 64) And then they connect this point to indistinguishability (a) above: "For exactly the same reason, one cannot in general determine, even retrospectively, whether discriminate or indiscriminate sampling was responsible for an actual sequence of historical events."
} 
say that it lands on 4 by chance and lands on 6 because of bias. By analogy, whatever the number of offspring of an individual, it makes no sense to ascribe it either to selection (if it matches the expectancy based on fitness as probability distribution) or to drift (if it does not).

This last version is the inscrutability of selection and drift strictly speaking. The indistinguishability in (c) concerns individual organism, the indistinguishability in (a) concerns token population, since it states that for a singular population we can't objectively say whether selection or drift account for its end state; and the indistinguishability in (b) concerns "history" of token populations and insists on our impossibility to disentangle several factors driving their evolution. The reasons that justify them are distinct: inscrutability (c) is established by general considerations on probabilities, as attested by the use of coin examples; these considerations also support indistinguishability (b); but the latter also is established by considering the effects of context-dependence when one models a population of alleles or organisms in a definite environment.

In this paper I consider and assess the three kinds of indistinguishability and their implications for the interpretation of fitness, selection and evolution. Indistinguishability may indeed raise issues for evolutionary biology: several research programs do presuppose that selection and drift objectively differ, and accordingly some alleles are selected and some go to fixation because of drift. For instance, at the genetic level, molecular biologists scan the genome and identify sequences under selection (selection vs. drift tests, e.g. Kreitmann test etc.) In another context and at the level of organisms, behavioral ecologists want to pick out traits due to selection, because by definition (Brandon 1990; Sober 1984) these are the adaptations. Thus, given that one main explanatory focus of the theory of evolution by natural selection is adaptation, the cost of statisticalism is to lose the explanatory goal of the theory (Bouchard and Rosenberg 2004). Granted, statiscalists argue that since they formally distinguish between drift and selection as what is and what is not predicted by trait fitness, their view "preserves the presumed explanatory relation between selection and drift" (Walsh et al. 2002, 465), even though this formal distinction can't justify making an objective distinction. But statisticalists fail to account for the way adaptation is explained, since the identity between evolution and natural selection would imply that any result of evolution (qua natural selection) is an adaptation, which is absurd. Finally, according to classical population genetics, any deviation from the expected Hardy-Weinberg equilibrium is due to selection and drift combined with different intensities. ${ }^{4}$ Various kinds of models uncover different cases of compositions, and as I previously mentioned Fisher's initial population genetic project consisted in the quantification of the relative contributions (of selection, drift, migration etc.) to specific evolutionary dynamics.

\footnotetext{
${ }^{4}$ For example: "While there may be cases of drift processes acting alone or selection processes acting alone, most cases of molecular evolution are best explained in terms of combinations of processes of drift and selection" (Dietrich 2013).
} 
Thus, we are left with the following problem: How to conciliate these common scientific views with the inscrutability of selection as promulgated by the statistical view?

\subsection{Preliminary Clarification: Fitness, Bias and Dice}

Fitness is a complex and somewhat controversial concept within evolutionary biology and the philosophy of biology. Thus one would wish to be as neutral as possible regarding fitness.

In the first basic uncontroversial sense of fitness, organismal fitness is the probability distribution of the offspring of an individual organism, and possibly its expectancy (often the other statistical moments are much less important ${ }^{5}$ ). As Orr (2009) states, this is an ecological property. Additionally given that within evolutionary biology we are typically interested in one or two loci (population genetics) or traits (quantitative genetics) we secondarily define the trait fitness, which is the "summary statistics" (Orr 2009) of all individuals having trait/allele $\mathrm{X},{ }^{6}$ that is, the averaging of organismic fitnesses of individuals in the class defined by the having $\mathrm{X}$. As previously noted one of the key claims of statisticalism is that such trait fitness is what is genuinely predictive within evolutionary biology (obviously we are not interested in a single individual), while also maintaining that trait fitness is just a statistical summary and not a causal property. ${ }^{7}$

Claims about the probabilistic nature of trait fitness and its consequences often employ parallels between coins or dices, and evolution. If a dice is biased, the value of the bias is what would correspond to organismal fitness in evolutionary biology, and is characterized in probabilistic terms. Trait fitness of a given trait value would then be analogous to the average probability that a dice, randomly taken from a set of dices (each with a proper bias value regarding side $j$ ), rolls on side $j$ (the side of the dice corresponding to the trait value).

A bias however always has some type of physical cause(s), such as a load in the dice, a magnet perturbing the roll, a specific habit of rolling the dice by the croupier etc. In the same way, the relative fitness advantage of an individual has a cause, which is the specific interaction between its traits and the environment; for example, the maximal running speed that allows a fast gazelle to escape its predators more effectively than other slower gazelles (the differences in maximal running speed, in turn, likely involving various anatomical, physiological and biochemical factors). This is important because the difference between fitness values and causes of fitness have been overlooked in the discussion of this topic. Yet they correspond to two

\footnotetext{
${ }^{5}$ Even though the relevance of other statistical moments such as variance or skew have been emphasised, first by Gillespie, and then by many authors who criticised the propensity interpretation of fitness (e.g. Ariew and Lewontin 2004, etc.). This does not really matter for the present argument.

6 Actually, Matthen and Ariew (2002) mainly argue that there are two concepts of fitness, the mathematical one and the ecological one (and this grossly matches the distinction I make, after Orr, between organismal fitness and summary statistics) and that they don't coincide. However while many geneticists can grant that, as I'm doing, few of them would follow the statisticalist conclusion. This paper argues that such understanding of fitness does not support the radical indistinguishability claims.

7 But for a critique of this role of trait fitness, see Ramsey 2013.
} 
different explanatory projects, one about the explanation of the process of selection ("which traits are going to fixation and at what rate, etc.?") and the other about the reasons why some traits in nature are selected, i.e. mostly the ecological question of the nature of adaptations. The first project can be completed by population geneticists by using only the fitness variable $\mathrm{W}$ and never trying to uncover the causes of $\mathrm{W}$ (since the genetic models abstract away from any ecological settings). The second task is undertaken by e.g. behavioral ecology, or paleontology (asking for instance "why had the Parasoraulophus such a long and hollow horn?"). The present paper will address issues of the inscrutability of the difference between drift and selection by always taking into account this multiplicity of explanatory projects related to evolutionary change and the process of natural selection. ${ }^{8}$

At this stage it is therefore useful to further probe in finer detail the problem that is raised by statisticalism about explanation. Trait fitnesses are the product of how traits (and trait values) contribute to the survival and reproduction of their carriers. Statisticalists conceive of natural selection as changes which are predicted (but not caused) by trait fitness, and for this reason they still make a difference between selection and drift, even though this distinction has no causal basis. But for this reason the claim that fitness is a statistical summary of interactions is not enough to account for evolutionary explanations: even if trait fitness is predictive, it does not say why some types of individuals will outcompete or reproduce more than others, resulting in the evolution of traits. Suppose indeed that two traits are always related, e.g. God paints all slow gazelles in red and all fast gazelles in green. Then the trait fitness of 'green' (or 'red') will be as predictive as the trait fitnesses of 'fast' (or 'slow'). Each will be in this example as good as the other when used in the Price equation. Sober (1984) famously distinguished selection-for and selection-of here: there is selection of green (because green gazelles are sorted against red gazelles) but selection for rapidity (and in turn whatever functionally facilitates that greater rapidity-biomechanical differences etc.). And a simple way to verify this is to counterfactually think of a world where fast gazelles are not green, etc., and reciprocally: we see immediately that there would still be selection for being fast, but no more selection of green. This is why Shapiro and Sober (2007) comment "Selection for is where the causal action is" (contra the statisticalists), because even if trait fitnesses are predictive the cause of evolutionary change takes place here-at the level of selection-for a property.

\footnotetext{
${ }^{8}$ Overlooking this multiplicity, the discussions between the papers by Walsh, Matthen, Ariew and Lewens, and Bouchard and Rosenberg (2004) seems to go nowhere since they talk past each others. Matthen et al. seem to focus on the selection process as it is investigated by population genetics, while Bouchard and Rosenberg focus on the ecological aspect.

More generally, given the multiplicity of explanatory projects, the aforementioned adaptation-centered critique against statisticalism might seem unfair at this point. Suppose indeed that we have a theoretical population genetics model of an abstract trait $\mathrm{X}$ with several genotypes distributed on two loci supporting the trait, and abstract fitness values. In this model, genotypic fitness or trait fitness is not related to any cause (the whole ecology is exogenous to the model). But the model is fairly predictive (regarding the end-states of the evolutionary dynamics that are modeled). It makes therefore no sense to criticize the fact that any possible adaptation can not be explained here because this is not part of the explanatory project.
} 
In the next section I will discuss a concept of natural selection that integrates this insight. I will then show how the distinction between selection and drift can be understood and justified against statisticalist objections.

\section{Concepts and Models of Natural Selection and Random Genetic Drift}

\subsection{The Conceptual Difference}

Firstly I argue that there is a conceptual difference between selection and drift. In this argument I utilize the framework of a difference-making account of causation applied to selection, which is shared by several recent views about selection (see Huneman 2012, 2013; Millstein 2006; Glennan 2009; Riesman and Forber 2005).

The difference-making (DM) account of causation views "A causes B" as "A makes a difference to B" (A being a variable, a property, a fact, etc.). Differencemaking can be cashed out in several ways, e.g. in counterfactual terms (Lewis 1973; Collins et al. 2004) or in manipulationnist terms (Woodward 2003). Applied to the causal nature of selection, selection for a trait (recall my previous example of gazelles with green/red and fast/slow traits) occurs when the nature of a trait makes a difference to the frequency of this trait. This view provides a simple distinction between selection and drift: when drift occurs, the nature of the trait does not make a difference for the change in its frequency. That is, if the trait had been different, this would not have (on average) changed the events accounting for the survival and reproductive success of organisms carrying it. Otherwise, the change in the final frequency of the trait would have been due to a change in reproductive success of the types of organisms, so the nature of the type of organisms (i.e. the value or nature of the trait) would have made a difference to the trait frequency, and this pertains precisely to natural selection.

Millstein (2002) draws a contrast between drift and selection in terms of the distinction between indiscriminate and discriminate sampling: for her selection involves discriminate sampling and drift involves indiscriminate selection, where here "discriminate" (resp. indiscriminate) means that the nature of the trait is relevant in that the sampling depends (resp. does not depend) on the trait value. Riesman and Forber (2005) and Shapiro and Sober (2007) argue for a manipulationnist approach whilst Huneman (2012) and Glennan (2009) argue for a counterfactual approach. The manipulationnist approach seems to be more affected by objections based on the notion of Gillepsie fitness, i.e. the fitness defined by the combination of expectancy and variance of offspring (in this definition, population size - and accordingly drift_-and selection are not independently manipulable) (Walsh 2010, forthcoming).

In the counterfactual approach, variation (e.g. increase) in frequency of traits (when there is selection) is counterfactually dependent on what traits are. When traits are fixed because of drift, their increase in frequency is not counterfactually dependent on what they are. To illustrate it, suppose that there are two types of eyes, blue and brown, which do not make any difference in reproductive success. However after a few generations, only blue eyes are found. This is a case of drift; it 
could equally be the brown or the blue eyes that went to fixation. So being blue does not make a difference to the fixation. Suppose now an alternative scenario in which females are more attracted to blue-eyed individuals, and blue eyes go to fixation. Then one would say that here blue eyes have been selected, and the justification is that it was precisely being blue-eyed that was, in some way, the reason for a higher reproduction rate via the female choice. Which means that this trait going to fixation in the population was (ceteris paribus) counterfactually dependent on the eyes being blue. The counterfactual relation more precisely holds between being blue-eyed and the reproductive success of organisms, and-assuming that blue eye is a heritable trait so that the offspring of blue eyes individuals tend to be more blue-eyed that the average - the frequency of blue eyes. It could be formulated as: "if the trait value (blue) had been different, the probabilities attached to reproductive success of their carriers would have been different", which entails different probabilities for their actual frequencies, and then for the average actual frequency of the class of the type "blue-eyed individuals". Thus these probabilities in the counterfactual statement have to be understood mostly as long-run propensities, and finally the probabilities present in fitness may be seen as long-run propensity (see Berkovitz, this issue, for this interpretation).

Thereby, when there are differences in fitness, and then one trait get fixed, one could look for the causes of fitness, just as one looks for the causes of a bias in a dice. These causes consist in the whole situation made up by the trait and its usual environment; that is, there is a specific robust relation between the trait and its environment expressible as a "reliable relation", which accounts for the fact that some value of this trait very likely overreproduces its competitors. For example, a maximal running speed may be such that the runners with this speed can run faster than their predators, so that other slower conspecifics are more likely to be eaten instead of them and thus leave on average less offspring. Natural selection means that there exists this reliable causal connection between trait and environment, which accounts for average differential rates of death and birth of organisms having various values of the trait-in the sense that, given a value of the trait, average rates of death and birth differ (e.g. the faster runners survive for longer on the average). In the case of fixation or elimination of a trait or an allele by drift, this is not accounted for by such reliable causal connection. Of course in this case there are still many possible causes of death of organisms taken individually, but these do not constitute causes of differential death/birth rates of organisms (regarding their values of a trait) that would be reliably occurring in the environment.

When there is selection for a trait, it means that the trait makes a difference to its increase in frequency, namely that had it had another value (e.g. decreased running speed), the result of the reliable connection abovementioned would be very likely to have differed, and then the average offspring number would probably have differed (e.g. the predators would have eaten more of the organisms under focus). This could be explicated in terms of possible worlds and then of counterfactuals: the closest possible worlds are the ones where the reliable factors (i.e. these environmental factors involved in the abovementioned reliable connection) still occur, so the value of the trait being different in these worlds predictably changes the resulting offspring number in most of these worlds. In the case of a trait or an allele being 
present because of drift, suppose that we consider versions of the evolutionary dynamics in the closest possible worlds: given that the reliable factors are still there, the initial trait value being different in these closest possible worlds would not yield a general determinate and different result that is highly probable across these putative possible worlds (Huneman 2012). (Here again the probability can be understood in terms of long-run propensity.) This is in essence the counterfactual version of the DM account, which I merely use here. ${ }^{9}$

The very nature of drift however has been discussed less extensively than the nature of selection. As Plutynski (2007) discusses, biologists hesitated between defining drift as those results that do not match the expectations of fitness and alternatively seeing drift as a set of processes whose direction is not definite and then seems random; they began by considering that random gamete sampling defines such a random process, and later on they included within the definition of drift as a process other ecological features such as founder effect, etc. Drift is a "catchphrase" as Plutynski says, and we note that some biologists (Gillespie 2004) even contest the idea that all the stochastic aspects of evolutionary processes are captured by this concept of drift. But in the present context, this has little significance because the distinction between causal relevance and causal irrelevance of traits to the frequency of types of organisms is robust to whatever the precise idea of drift one supports.

Now suppose that, following on from my example, "brown eyes" are fitter. In a small population, it may happen that "blue eyes" still become fixed: random deletion of brown eyes occurs ("random", in the sense that being brown plays no role here, and not in the sense of assuming that the factors causing alleles for brown or blue eyes are intrinsically stochastic) and vice versa; and eventually blue eyes are fixed. In a large population, this blue/brown ratio of random deletions is less likely to be the case, because the two kinds of random deletions will probably compensate, so that what remains is the higher number of brown eyes due to the effects that reliably led to higher chances of reproductive success for brown-eyed individuals when compared to blue-eyed organisms. ${ }^{10}$ Therefore, the larger the population the higher the chances that, if there is selection for brown eyes, no random deletion of brown-eyed individuals will prevent selection from driving this trait-value to fixation. It therefore appears that the conceptual difference between drift and selection entails properties that are common to all mathematical models of population genetics (Fisher-Wright models, other one-locus models like Moran model, two loci models, etc.), namely the relation between population size and drift, according to which drift can be understood as demographic stochasticity—as I detail it now.

In effect, in a Fisher-Wright model drift is explicitly modeled as a binomial law. For a population of size $\mathrm{N}$ with two types of alleles and no selection, in such model one demonstrates that the probability that two randomly taken alleles (with initial

\footnotetext{
${ }^{9}$ It is defended in Huneman (2012) - which specifies the account for different types of selection (i.e. directional, balancing, stabilising) and considers some objections; see Huneman (2013).

${ }^{10}$ Beatty put it in these terms, in his discussion of the difference between selection and drift: "in an infinite population, any local misrepresentation of gene and genotype frequencies due to indeterminate sampling will cancel out." (Beatty 1994, 205).
} 
frequency $\mathrm{p}$ and $1-\mathrm{p}$ ) are different is given by $\mathrm{H}_{\mathrm{t}}=\mathrm{H}_{0}(1-1 / 2 \mathrm{~N})^{\mathrm{t}}$ [with $\mathrm{H}_{0}$ being this initial probability $\left.\mathrm{H}_{0}=\mathrm{p}(1-\mathrm{p})\right]$. This entails that when the population is small ( $\mathrm{N}$ small), the probability of having different alleles in the population tends very quickly toward $0(1-1 / 2 \mathrm{~N}$ is much closer to 0 than when $\mathrm{N}$ is large, so the series converges more quickly to 0 ). Which means that after few generations all alleles are very likely to be almost the same: hence one of the allele types (indiscriminately) is probably lost in the population. When $\mathrm{N}$ is large this probability is very slowly decreasing since for a long time it is close to $\mathrm{H}_{0}$ (because $1 / 2 \mathrm{~N}$ is very close to 0 ), which means that very probably both alleles are maintained within the population for a long time (Gillespie 2004, 26); hence, the larger the population the smaller the drift effect.

Notice that often "drift explains fixation of X" (X being the brown allele in my example) means that $X$ "is less fit but goes to fixation" $(\alpha)$. This is what considered the Fisher-Wright model I just mentioned. However, Matthen (2009) emphasized that " $\mathrm{X}$ is here by drift" may also mean " $\mathrm{X}$ and $\mathrm{X}^{\prime}$ are equally fit but $\mathrm{X}$ (resp. $\mathrm{X}^{\prime}$ ) goes to fixation" $(\beta)$. Yet the two cases of drift are explained exactly in the same way: the smaller the population the higher the chances that there will be no compensation between opposite random effects deleting instances of the traits. When the fitnesses of brown and blue are equal, and the population is small, the chances are high that there will be enough random deletions of brown to drive the brown allele to extinction, which is case $(\beta)$. ( $\beta$ ) appears therefore as a limiting case of $(\alpha)$, when the fitness of $X$ tends towards the fitness of $X^{\prime}$.

\subsection{Sequences of Populations: "Ecological Selection" and the Token-Type Difference}

The DM account emphasizes the close relation between process of selection and the aforementioned "reliable factors"; as we saw, the properties of random genetic drift rely on the different chances that these factors have indeed their predictable effect on traits frequency - these chances being conditioned by population size. Note that the nature of these "chances" have been left unaccounted. However, the reasoning here is not different from any other reasoning that uses the weak law of large numbers, and draws consequences from the mathematical fact that frequencies converge (in probability) towards chances. No specific interpretation of probability is required here. ${ }^{11}$ The above reasoning does not depend on whether chances are propensities, or the world is deterministic and chances refer to some objective probabilities that are compatible with determinism, or even whether chance refers to some subjective probability that is based on objective facts.

Using the (weak) law of large numbers also allows one to make some distinctions between facts of selection and facts of drift, if we stop considering the level of individual organisms (as is considered in inscrutability claim c) and switch to the level of populations.

\footnotetext{
11 I used here long-run propensity interpretation for the chances that are mentioned in the counterfactual statements. But here I consider the probabilities of different effects by the "reliable factors" in this world, which is different.
} 
First, suppose that we repeat experiments of selection in a population of size $n$, where traits $\mathrm{X}$ and $\mathrm{X}^{\prime}$ have fitnesses $\mathrm{W}$ and $\mathrm{W}^{\prime}\left(\mathrm{W}>\mathrm{W}^{\prime}\right)$, respectively. Then, $\mathrm{X}$ has a higher chance than $\mathrm{X}^{\prime}$ to go to fixation. The analogy with dices would be a dice biased toward " 6 ": one series of rolls may not show more incidents of " 6 " than other sides, but in a sufficiently large set of series of rolls we could expect to get more series where the relative frequency of " 6 " is higher than $1 / 6$. Hence, if $X$ 's chance to leave more offspring are higher than $\mathrm{X}^{\prime}$ 's then in a sufficiently large number of repeated experiments/simulations, we should expect to see more often the outcome $\mathrm{X}$ than $\mathrm{X}^{\prime}$,

Inversely, if drift alone is acting (i.e. $\mathrm{W}=\mathrm{W}^{\prime}$ ) no outcome is more likely than another, then it is very likely that there will be no obvious pattern of overfixation in favor of one or another trait. This is the typical picture of drift and selection in repeated runs (Fig. 1).

Now suppose that we have an experimental apparatus allowing us to change the population size while keeping fitnesses constant. Suppose we have alleles $\mathrm{X}$ and $\mathrm{X}^{\prime}$ (in all the following their initial frequencies are constant and, to make things easier, equal to one another). If one increases the size $\mathrm{n}$ of a population while keeping constant the relative fitnesses $\mathrm{W}(\mathrm{X})>\mathrm{W}\left(\mathrm{X}^{\prime}\right)$ and initial frequencies, then we witness the following pattern:

(S) The outcome of the dynamics will be more and more likely to be $\mathrm{X}$, or, in other words, the larger is $\mathrm{n}$, the higher are the chances that the outcomes in sets of populations of size over $n$ will be mostly the fixation of $\mathrm{X}$.

In the populations where $X$ does not get fixed, then we would say that $X^{\prime}$ is fixed in the population by drift, in the sense $(\alpha)$ of drift above (see Fig. 2; K represents one such population). The fitness difference $\mathrm{D}=\mathrm{W}(\mathrm{X})-\mathrm{W}\left(\mathrm{X}^{\prime}\right)$ determines probabilistic properties of this series, both regarding the distribution of populations where $X$ fixes and where $X^{\prime}$ fixes, and the speed at which fixation takes place. The larger is $\mathrm{D}$ (or in other contexts what is called $\mathrm{s}$, the selection differential), the lower the chances that there will be populations where $\mathrm{X}$ does not get fixed, and the higher the chances that in a given period of time $\mathrm{X}$ will have gone to fixation in a given population.

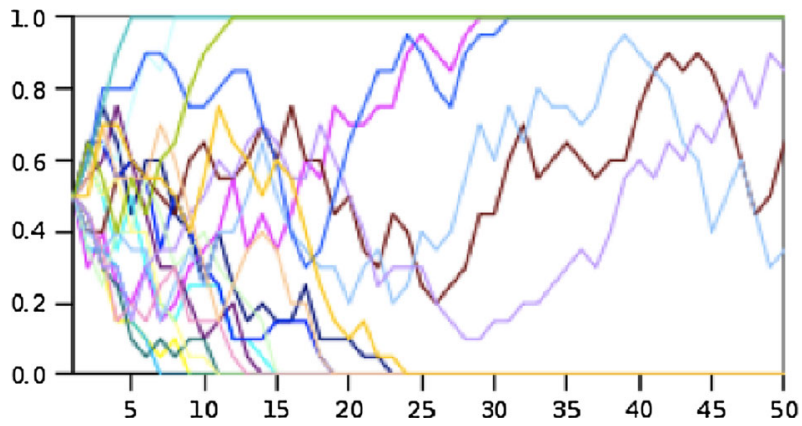

Fig. 1 Fixation of $\mathrm{X}$ or $\mathrm{X}^{\prime}$ in repeated runs $\left(\mathrm{W}=\mathrm{W}^{\prime}\right.$ : random genetic drift alone). Vertical axis frequency of $\mathrm{X}$, horizontal axis number of generations 


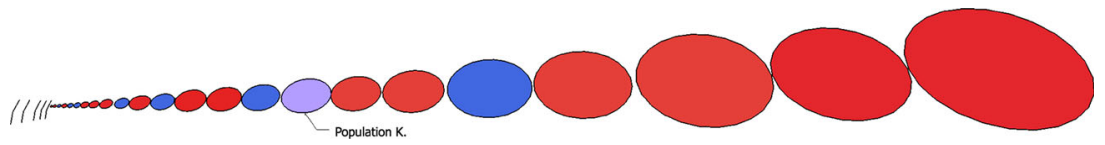

Fig. 2 Repeated experiments of evolution with $\mathrm{W}>\mathrm{W}^{\prime}$. Pattern showing "Ecological Selection" and random genetic drift fixing $\mathrm{X}^{\prime}$ in token populations like $\mathrm{K}$. (In red populations where $\mathrm{X}$ goes to fixation; in blue, $\mathrm{X}^{\prime}$ goes to fixation. The size of the population is represented by the radius of the circles.). (Color figure online)

So clearly in this sequence there is no inscrutability at the level of populations: some token populations are driven by drift, others are driven by natural selection. And when we consider a theoretical model in population genetics, saying that selection is acting in a population implies, in turn, that if we were to make this ceteris paribus iteration of population with increasing $n$, we would witness this exact pattern of fixations as a function of population size, which can be labeled the "statistical signature of selection". Indistinguishability (a) and (b) do not seem to occur. And indeed nothing here seems to differ from any case where science deals with a process that involves some level of noise and fluctuations, so that in some cases a process will not yield its expected result but the overall trend/pattern across cases would be as expected.

The confusions here about inscrutability arise from the fact that there are two different things people consider: the token populations, and the sets of hypothetical populations. In one population where $\mathrm{X}^{\prime}$ gets fixed, drift has been acting (it is represented by population $\mathrm{K}$ in Fig. 2). But the series of population demonstrates that in this case although the fixing of $\mathrm{X}^{\prime}$ is due to drift such kind of populations characteristically are driven by natural selection, or in other words, the population is such that reliable factors underlying natural selection for a trait are taking place which is what we could call "ecological selection". In the case where $\mathrm{X}^{\prime}$ gets fixed, we could say that the token population doesn't mirror the ecological selection taking place in this type of population. We could see an analogy with dices in the following example : suppose a set of series of rolls, such that in the large majority of them the frequency of side " 6 " is $1 / 3$. We would then say that there is a bias, and that the dice is loaded. But among the set there might be series in which the frequency of 6 is indeed 1/6; we would not say that the dice stopped being loaded in such series, but rather that some sort of "sampling error" occurred within them, in the sense of some events that increased the frequencies of rolls of the faces ' 1 '- '5': variations in some of the initial conditions that are too fine-grained to be represented in the model of dice rolls, or the momentum of the roll, etc., even though the bias still exists as a factor that determines the expectation of the frequency of ' 6 '. "Drift" in biology is the (catchphrase) name for such a sampling error. Saying that drift explains an outcome in a token population means the same thing as saying that sampling error in this series produced the outcome. So it does not mean that no selection occurred at all, but it indicates that some "ecological selection" occurs yet its effect had been swamped, as most biologists would intuitively say, meaning that there are factors that are responsible for selection and there are other factors that are not considered part of the selection, and drift is constituted by cases when the factors that are not considered as part of the selection dominate (although the factors that are 
responsible for selection are still operating). "Ecological selection" is the analogon of the load in cases when even though there is a load in a dice, in a series of rolls of the same dice, the dice still mostly rolls on the side that is not loaded. The set of hypothetical populations informs us about "ecological selection" as well as the set of series of rolls informs us about the loadedness of the dice, because both allow to infer the biases or the fitnesses on the basis of frequencies.

But what about cases of drift in the sense of $(\beta)$, namely, when $W=W^{\prime}$, i.e. situations of "drift alone", analogous to situations where an unloaded dice rolls (probabilities of all faces are equal)? Let's consider now a series of sets of duplicate populations of size $n$. As we saw, drift correlates with population size, namely, the smaller the population size, the higher the chances that indeed some random events will favor the fixation of one trait type rather than the other. So, reciprocally (writing $Y_{n}$ the number of occurrences of n-size populations where $X$ and $X^{\prime}$ both get fixed) $Y_{n}$ will increase with $n$. On the other hand given that there is no reason for $\mathrm{X}$ to be fixed rather than for $\mathrm{X}^{\prime}$, and that this is not dependent upon the population size, the ratio $Z_{n}$ of populations where only $X$ gets fixed does not change in a monotonous way when $\mathrm{n}$ increases; but it tends towards 0 when $\mathrm{n}$ becomes infinite since at the limit we lose neither $\mathrm{X}$ nor $\mathrm{X}^{\prime}$. This statistical pattern, which is the "statistical signature of drift alone", is therefore the following:

$\left(S^{\prime}\right)$ increase of $Y_{n}$ in probability, $Z_{n}$ tends towards 0 in probability.

According to this pattern, in all cases where one of those traits gets fixed and not the other in a token population, we would say that $\mathrm{X}$, or $\mathrm{X}^{\prime}$, is here by random genetic drift. (See Fig. 3) But in this type of population there is indeed no ecological

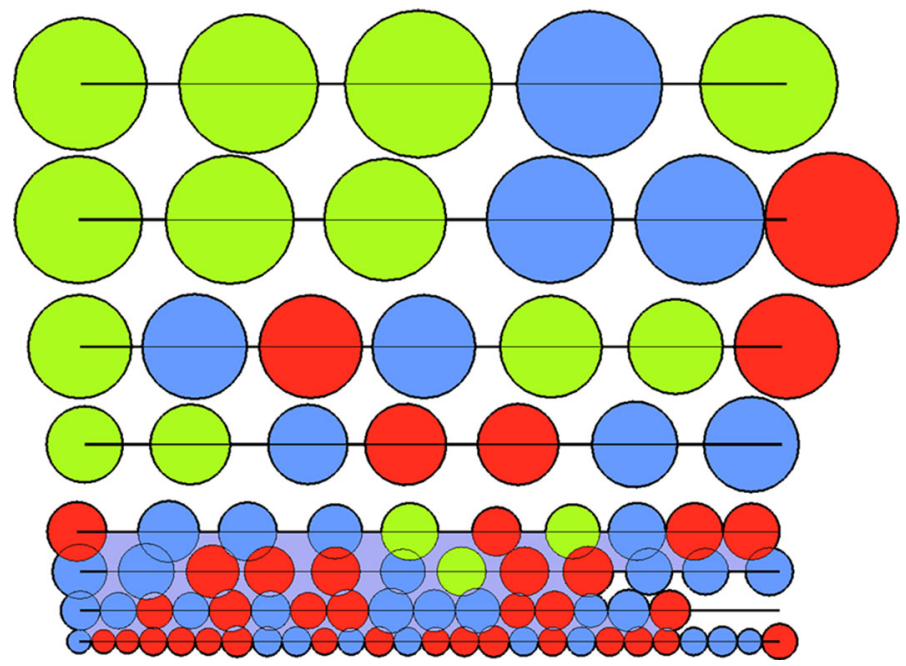

Fig. 3 Statistical signature of random genetic drift alone. The radius is the size of the population; each line is a set of same size populations (after 10,000 generations). Blue populations: $\mathrm{X}$ fixes; red populations : $\mathrm{X}^{\prime}$ fixes; green populations: $\mathrm{X}$ and $\mathrm{X}^{\prime}$, coexist their amount is noted $\mathrm{Y}_{\mathrm{n}}$ in the text. The series of sets of populations shows the signature of drift alone $\left(\mathrm{W}=\mathrm{W}^{\prime}\right)$ described in the text. (Color figure online) 
selection, since we don't witness the statistical pattern defined above that signals the differential effect of reliable factors.

Nevertheless the two senses of drift are not independent. Obviously, $\mathrm{W}=\mathrm{W}^{\prime}$ is the limit of case $(\alpha)$ when $\mathrm{W}-\mathrm{W}^{\prime}$ tends towards 0 (which is case $(\beta)$ ). When $\mathrm{W}>\mathrm{W}^{\prime}$, we will witness the signature of selection (Fig. 2), in which the $\left(\mathrm{X}^{\prime}\right)$ populations (meaning populations where $\mathrm{X}^{\prime}$ goes to fixation) are the only cases where we say that the fixed allele is here by drift. But when for a given population size $n$ this fitness difference decreases (in other words the selection for $\mathrm{X}$ gets weaker), the number of $\left(\mathrm{X}^{\prime}\right)$ increases in probability, so in order to very probably reach what was the former value of number of $(X) /$ number of $\left(\mathrm{X}^{\prime}\right)$ at $n$ one has to increase $n$. At the limit $\mathrm{W}=\mathrm{W}^{\prime}$, populations where both traits get fixed (stable polymorphism) appear, and we witness the signature $\left(S^{\prime}\right)$ proper to the case $(\beta)$ of drift.

\subsection{On Inscrutability}

Returning to our three senses of indistinguishability seen above (Sect. 2) indistinguishability (a) about the fact of selection and drift in token populations, indistinguishability (b) about their apportioning and inscrutability (c) about individual organisms as selected-, we see now that, even if the inscrutability (c) is clearly justified, the one concerning token populations (a \& b) is not so obvious, given that the consideration of a series of population may show ecological selection in the type of population and drift in some token populations. On the contrary these are not quite so inscrutable as soon as we think of them in series of populations instantiating what we could call a population type [think of bacterial evolution, such as Lenski and Travisano (1994) experiment]. Those statistical patterns described above define the signature of the fact of ecological selection. Whether a token population displays, or not, the typical outcome of ecological selection means whether selection or drift has been acting.

Conversely even if we don't know the fitnesses but can repeat duplicate experiments as in Sect. 3.2, we can decide whether selection or drift is acting because we can identify these different statistical signatures that indicate whether ecological selection is there in the population type and whether selection or drift is acting in a population token. This means that there is a difference in frequency patterns between drift (sense $\beta$ ) and selection (what I call statistical signature), which follows from their conceptual differentiation as described above. If we witness for instance a pattern where $Z_{n}$ increases, it will be a population where ecological selection acts (thus, token populations where $X^{\prime}$ gets fixed are driven by drift). If on the contrary, $Z_{n}$ does not show any directionality, we have a signature of drift of kind $(\beta)$ : no ecological selection for one trait occurs in this set of populations. And considering several sets of populations of various sizes allows one to attest the effect of drift, if such a pattern of outcomes is visible. ${ }^{12}$

\footnotetext{
12 This was done in Lewontin and Dunn (1960) experiments on the varieties of $t$ allele in mice. They varied the population size and have shown that drift is acting on the evolution of the $t$ allele because in smaller populations variance in the fixation of alleles $t$ and + is higher. "The effects of changing
} 
Millstein (2006) and Millstein et al. (2009) distinguished between "process" and "product" when we talk of selection and drift. Statistical patterns depicted here concern the product, since we look at what goes to fixation. However, considering the effect of varying population size in our samples of population also concerns the process of drift. Increasing the population size indeed requires more events to drive an allele to extinction. Therefore, the speed of fixation of one allele against the other decreases when population size increases. So statistical signatures, here, may also concern drift and selection as processes. Actually the same analysis could be done by considering dynamics and fixation rates instead of fixations. It would provide more precise distinctions between selection and drift but I won't enter this difficult analysis here.

The difference in signatures is what is often meant by saying that drift is demographic stochasticity and selection is deterministic or directional (e.g. Lenormand et al. 2008). Deciding whether the biological universe is deterministic, in the sense that each distribution of organisms at time t determines exactly the distribution of organisms at time $t+1$, is immaterial to the above distinction between selection and drift (even though it may be relevant for the question of the interpretation of the probabilities that are involved in the characterization of selection and drift). Selection is said to be "deterministic" in the sense that our populations of increasing size tend towards a limit where the initial state of the population determines exactly the final state of fixation, and this holds even if the biological universe is "indeterministic" in the sense I just used. (However, cases of both stabilizing and disruptive selection can also be said to be 'directional' in the same way, because in the sets of populations with increasing size that would correspond to such cases, they would display specific trends for the variables $Z_{n}$ and $Y_{n}$, analogous to what was seen with directional selection strictly speaking). For this same reason "ecological selection" can in general be called "directional" in this sense too because this series of population of increasing size tends towards a specific state of fixation. Random genetic drift is stochastic in the sense that with "drift alone" this is not the case.

One more general lesson to draw from the above considerations concerning frequencies in samples of populations is the following: for a given class of populations of a given population size $n$, the ratio $Z_{n}$ can be modified by acting on two variables-either $n$, or $\mathrm{D}=\mathrm{W}-\mathrm{W}^{\prime}$. Increasing $\mathrm{n}$ means that we increase $\mathrm{Z}_{\mathrm{n}}$, as we have seen. But the same result can be reached by increasing $\mathrm{D}$, because this means increasing the intensity of selection, and thereby the number of (X) will increase. So the comparative rates of fixation of $\mathrm{X}$ and $\mathrm{X}^{\prime}$ in samples of population are modified by alternatively acting on these two variables: fitness difference and population size. ${ }^{13}$ To put it in a different way: if in a class of same-size populations the ratio $(\mathrm{X}) /\left(\mathrm{X}^{\prime}\right)$ varies, in the absence of further information there is no way to ascribe this change to either of these variables.

\section{Footnote 12 continued}

population size are highly significant (...) a smaller population size causes greater genetic drift both toward lower and higher frequency of the + allele".

13 The problem of non-independent interventions, that Walsh (2007) took from Gillespie's work, is not crucial here. However Walsh's argument against causal variables is addressed in Huneman (2013), and Abrams (2013) has an argument similar to what could be derived from the present paper. 
If we also consider the dynamics of two trait values $\mathrm{X}$ and $\mathrm{X}^{\prime}$ (not alleles), the complete picture should at least include another variable, i.e. heritability. Keeping a fixed fitness difference and population size, decreasing heritability $\mathrm{h}^{2}$ will indeed decrease the response to selection and has the same effect as decreasing D (and vice versa). It means that if we plot $Z_{n}$ in a graph there should be four dimensions: $D, n$ and $h^{2}$, and the value $Z_{n}$ on the fourth axis can be the result of a set of combinations of values of these variables. Therefore what is meant by the idea that selection, drift etc., are forces acting on populations is only that the possible sets of outcomes in population series of a population type are determined by these three variables. But I leave this extra complexity aside and only consider our samples at a fixed value of heritability and initial allelic frequencies.

Granted, considering frequency patterns allows one to identify drift and selection as distinct outcomes in samples (or sets) of populations. But what about considering only one population with no access to a sample of populations-which is of course the case when scientists investigate selection in the field (e.g. Endler 1986)? Here is where we face the problem of what I call opacity.

\section{The Epistemic Opacity of Natural Selection and Random Genetic Drift}

Here I show that there is a different type of opacity from the metaphysical inscrutability pinpointed by Matthen, Walsh and Ariew, and that is essentially epistemic. Regarding my distinctions in indistinguishablity (Sect. 2), it is about the kinds (a) and (b) that concern token populations, not individual organisms.

\subsection{DM Account, Reliable Factors and Epistemic Opacity}

The question of the distinction between selection and drift concerns whether there is a fact of the matter to decide the question "Is it selection or drift which is responsible for the fixation of X?" The inscrutability shown by the statisticalists excludes that such fact of the matter could be a difference in the individual (organisms) we are considering, but this does not preclude any other kind of fact of the matter. Recall that selection entails that there has been some factors, that I will now call selective pressures, which occur in a wide portion of possible worlds close to ours, and which are therefore reliable factors. Those factors constitute what I defined as "ecological selection", whose occurrence can be attested when considering series of sets of population with increasing size. Other factors can be non-occurring in those close worlds (such as the results of gamete samplings, some unusual predators, etc.), and we ascribe to random genetic drift their effects on population in this world (Huneman 2012, 2013). So concerning natural selection there is metaphysically speaking a fact of the matter in the set of possible worlds (e.g. Woodward 1999), in the sense that the distribution of these factors across worlds is a fact even though we may not be aware of it. This means that, to the extent that an indistinguishability between selection and random genetic drift 
concerns the distribution of these factors in a token population, it will be an epistemic one.

Regarding the difference made on the outset of this paper between explanations of natural selection in actual ecological settings and population genetics models of natural selection, an additional distinction should be made between two kinds of questions: the question of whether and why natural selection occurred in an extant population of organisms (1), and the question concerning population genetics models and outcomes of runs of simulated evolution (2). The present considerations about counterfactual dependence and reliable factors do establish that there is such a fact of the matter for question (1). Yet about the question (2), since we only assume fitness values and then design models to see which possible evolution may happen, these facts of the matter may not be defined. The fitnesses are defined in the model, but nothing within the model will decide whether fitness values of traits or alleles are the "true" ones found in nature. In the context of population genetics models per se there is of course no problem of any limited access to the fact of the matter concerning counterfactual dependences, reliable factors, etc.

The question of applying a given (population genetics) model to understand a specific case is therefore the question of whether the ecological facts (defining the abovementioned reliable factors) confer to the various traits or alleles the fitnesses values and the fitness differences that we ascribed to them in the model intended to explain the case. Therefore the question of the decidability of selection versus drift now touches upon a fact of the matter concerning this relationship between ecological facts and fitness values. Thus the previous considerations about limited epistemic access do hold.

Indeed in many cases we may not know which are the reliable factors and which are not, so we may not, even in principle, be able to tell natural selection from drift. Yet this is an epistemic impossibility: it is not due to the metaphysics of evolution, as it was in the case of inscrutability (sensu (c)) regarding organisms. I call it an "epistemic opacity". It is pervasive for the following reason. Suppose a population with some organisms that for trait $\mathrm{x}$ have the value $\mathrm{X}$ and other organisms having the value $\mathrm{X}^{\prime}$ (to simplify $\mathrm{I}$ call these trait $\mathrm{X}$ and trait $\mathrm{X}^{\prime}$ organisms below). $\mathrm{X}$ individuals reproduce more successfully than $X^{\prime}$ individuals. To say that it is because of selection, hence to confirm the counterfactual statement about the specific value of $\mathrm{x}$ and the increase in frequency of some kind of organisms, we should be able to connect the greater reproductive success of $\mathrm{X}$ organisms to the existence of reliable factors. Namely factors that would still be there in possible worlds close to ours and would be responsible of different trait frequencies in worlds where the value of $\mathrm{x}$ is differently distributed. Yet our knowledge of these factors supposes that we have some knowledge about the set of possible worlds that are the closest. This is not always the case and that is one of our obvious epistemic limitations.

One of the reasons for that follows from the fact that laws of nature are a major way for us to know of possible worlds: for instance, the laws of physics tell me that if I was to dig a hole in the bottom of my boat it would sink; hence in a possible world exactly like ours but where my boat has a hole in its bottom, this boat with the hole will sink. Because laws offer support to counterfactuals they are good 


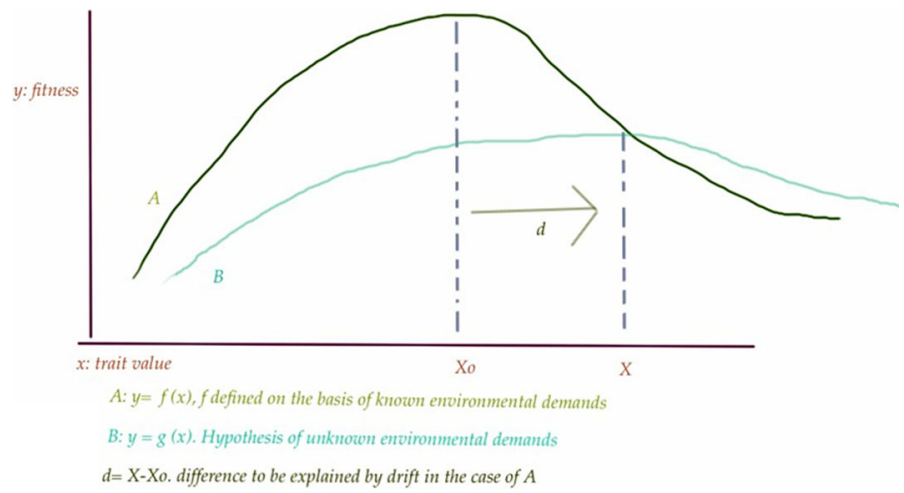

Fig. 4 Epistemic opacity and optimality modeling

indicators of what possible worlds are around ours. ${ }^{14}$ But given that we do not know all the laws of nature then clearly we do not always know what are the reliable factors that are constant across a set of possible worlds close to ours. Therefore we are not epistemically able to access the metaphysical facts of the matter that support the distinction between selection and drift in a given case.

Given that in a specific case we do not always know which laws are instantiated, even the knowledge of what are the laws of physics is not enough to allow us to know what happens in the closest possible worlds. For example, suppose that we know a croupier who is biased and makes a roulette more likely to hit the 0 . Then given our knowledge of laws of physics we would think that in the closest worlds where the croupier is replaced by a neutral one the roulette would be equiprobable. However, if it was also the case that this roulette is in a magnetic field and that the quantity of iron in each number of the roulette is different, then even if we had changed the croupier the roulette in the closest worlds so defined would still remain non-equiprobable. Therefore the knowledge of possible worlds close to ours relies both on the knowledge of laws of nature and on the knowledge of all the relevant facts in the actual situation.

Epistemic opacity can be illustrated by a situation in behavioral ecology, when a biologist cannot account for the fact that a population does not display the optimal value $X_{o}$ of a trait $x$, predicted by the environmental demands (Fig. 4). One could explain the difference $\left(\mathrm{X}-\mathrm{X}_{\mathrm{o}}\right)$ by the fact that the population is small and that there has been random genetic drift. This means that in other possible worlds close to ours the value of $\mathrm{x}$ is not $\mathrm{X}_{\mathrm{o}}$ but may be different from $\mathrm{X}$. But an alternative explanation would be that in our world there is another (as yet unknown) environmental demand at stake and if it is taken into account then the true optimum value is $\mathrm{X}_{\mathrm{o}}^{\prime}$ (which turns out to be much closer to $\mathrm{X}$ ). In this case in worlds closest to our world, since this selective pressure is also acting the value of $\mathrm{x}$ would not be $\mathrm{X}_{\mathrm{o}}$ but would still

\footnotetext{
14 In Sober's framework (see Sober 1984), one would say that we do not always all the source-laws according to which organisms behave in their ecological setting, which impinges on the consequencelaws that population genetics captures.
} 
be close to $X$ because it would be optimally fixed around $X^{\prime}{ }_{0}$. So now, the value of $\mathrm{X}$ would correctly be explained by natural selection (the selective pressures being both the known and the unknown factors) and not drift. ${ }^{15}$ If the population is small, a good test to discriminate between both hypotheses would be to consider a larger identical situation (ceteris paribus): if $\mathrm{X}$ tends now towards $\mathrm{X}_{\mathrm{o}}$ we would say that the deviation under consideration was a case of drift and not selection. Yet in a small population, but with no possibility to consider a larger replicate population, and given our incomplete knowledge of the ecology, the epistemic opacity is unavoidable.

Notice that this epistemic opacity is not absolutely proper to evolution by natural selection. It seems that in any cases when researchers hunt for complex causal facts they face an epistemic opacity of the same nature to the extent that these causal facts (as underpinned by counterfactual dependences) are underwritten by relations between possible worlds. However, even if I'm wrong on this it does not matter to the next point I want to make; which is that the small populations are much more prone to this epistemic opacity.

\subsection{Epistemic Opacity and Population Size}

According to many conceptions natural laws involve causal facts. In turn causation is often related to probabilities (e.g., causes increase probabilities of effects, or causal facts are indicated by probabilistic dependences etc.). And probabilities are related to frequencies through the (mathematical) weak law of large numbers. So the more you can observe relative frequencies in this world, the more you are likely to discern the laws of nature that are instantiated in a specific case, hence establish robust facts regarding possible worlds, and finally distinguish what is due to natural selection from what is due to drift. Therefore large populations are less affected by epistemic opacity than small populations. I will now develop these basic considerations in order to specify cases where epistemic opacity is robust.

Consider the parallel with a dice. Each possible outcome of a roll is due to both its structure or the structure of the settings and the peculiar circumstances of this roll. In the closest possible worlds you would change these singular circumstances and the overall result would change. But what concerns the structure of the dice and its setting across these worlds is constant. Suppose a series of 18 rolls in which " 6 " occurs half of the time. This does not allow you to say that " 6 " is loaded because you don't know whether this over-representation of " 6 " (compared to the default expectation, namely 3) is due to something general about the dice that concerns the structure of the settings (a load is such a thing), or to singular circumstances of each roll. However if you settle 15,000 rolls and " 6 " is over-represented you are entitled to think that there is plausibly either a load on 6 or some other cause of the bias. Effectively in the closest worlds it is merely a few singular circumstances that change. Thus in a long series these changes of few circumstances do not make a difference to the general pattern of outcomes and then to the overall frequencies.

\footnotetext{
15 Rosenberg (1994) pushes to the limit this reasoning and argues that in principle there is no drift, but only such cases of ignored selective pressures.
} 
Therefore, the fact that there is an important difference between the expected frequency of " 6 " given the default assumption (equiprobability) and the actual frequency indicates that we were wrong in specifying the world where we are (i.e. in assuming a default equiprobable setting). Hence the very long series of rolls indicates something about the facts that are recurrent in the closest possible worlds; namely there is something constant across those worlds that correlates to a higher frequency of "6".

Similarly, in biological evolution, if some trait $\mathrm{X}$ goes to fixation in a very large population then in general we conclude that there are selective pressures on this trait (or on correlated traits) and that it is under selection. The reasoning is analogous to the inference we make in the case of a large series of dice rolls. In both cases, dices and biology, other counterfactual tests could discriminate between causes and correlated effects (load and painting on face 6, or fur color and maximal running speed). Actually we sometimes have independent ways to consider whether the trait that increases in frequency was under selection, or correlated to a trait under selection, described in terms of 'selection for': if it is not possible to make counterfactual tests, such as the ones we would do to disentangle the load in the dice and the painting on the side of the dice, we can still turn to comparative biology (Harvey and Pagel 1991). That is, if trait $\mathrm{X}$ is such that in another species' population and similar environment trait $\mathrm{Y}$ also increases in frequency and trait $Y$ is very similar to trait $X$, this increases the likelihood that $\mathrm{X}$ is under selection (and not simply correlated to a trait A that is actually under selection-see Endler 1986) and Lande and Arnold (1983) for a formal treatment in quantitative genetics. This is to say that the epistemic opacity can be so overcome. Nonetheless disentangling correlated traits not under selection from traits under selection is possible because of comparative data. But it does not solve the question of disentangling the selective pressures themselves in order to decide for which pressure exactly trait $\mathrm{X}$ has been selected: this seems to be an opacity that can not be solved in principle (Lewens 2010). However, here I am only concerned with the opacity between natural selection and random genetic drift.

Now take a very small population (with perhaps even $<15$ individuals). There is no obvious way to establish whether some factor responsible for the differential survival/reproduction of various types of individuals is a reliable factor, because we have no clues about which are the closest possible worlds where organisms do not have trait X, exactly as in the case of our short series of dice rolls above. We may of course have a default expectation, that would be the analogon of the equiprobability for the case of the dice, but any departure from this expectation does not license us to infer anything. Knowing in more detail what happens in our small population does not change anything to this situation, except if by enlarging this knowledge we come to know more nomic connections (because they uncover reliable factors likely to occur in the closest possible worlds). This is what leads mistakenly to think that there is no fact of the matter to decide between selection and drift.

Therefore in small populations the epistemic opacity of selection and drift is pervasive. Whereas the inscrutability of selection and drift, concerning individuals (sensu (c)), was not tied to the size of the population and was a metaphysical fact (i.e. the meaninglessness of any question about who is selected) the opacity of selection and drift can be reduced (and it is the real meaning of indistinguishability 
sensu (b)). It is an epistemological feature often depending upon the size of the population. Here we are not dealing with the meaninglessness of some question but rather with the difficulty, in practice or in principle, of answering it.

\section{Objections to the Reducibility of Epistemic Opacity}

One way to bypass the epistemic opacity is, as I described, by the consideration of large populations. Yet there is a very simple possible objection here which I have to address. (Until now, the question of interpreting probabilities in evolutionary theory was not crucial. I used the notion of statistical signature to pinpoint properties that seem to be independent of the interpretation of probabilities. Yet from now on the question will have to be raised.)

\subsection{Issues Concerning the Inference from Large Populations}

Suppose that there is a very large population where a trait $\mathrm{X}$ increases in frequency and goes to fixation. Usually, according to Sect. 4.2, I would say that X is selected for and that there are causes of this process, namely, those reliable factors that put selective pressures onto organisms and that would be conserved across a specific range of possible worlds close to ours (exactly like in the case of the dice an overrepresentation of " 6 " when compared to the default expectation constituted by equiprobability would lead me to say that there is an effective cause somehow related to side "6", like a load, that accounts for the excess in its frequency). However, even if we have inferred that $\mathrm{X}$ has a higher fitness, it is still possible that the actual changes in the observed frequencies of traits are not causally related to the putative causes of fitness (in the sense of these reliable factors mentioned above). In other words, it can be the case that even if there is ecological selection in the population type, in a token population the outcome is not due to the reliable ecological factors underlying the causal relevance of one trait value to its frequency fate. This can mean two possible things, defining the two problematic cases faced by any inference from " $\mathrm{X}$ is fixed in a large population" to " $\mathrm{X}$ has been selected". Both problematic cases are objections to such an inference and are addressed now.

\subsubsection{Problematic Case \#1}

If we independently define the selective pressures (the reliable factors) and compute the ability of each organism type to cope with them, or if we define directly the fitness values (e.g. when setting the parameters of a simulation in population genetics) and if the traits subsequently going to fixation are not the ones that best face, on the average, the selective pressures, or have the highest fitness, then it is random genetic drift and not selection that leads them to fixation. It's exactly one of these cases (like population K) of "here by drift" in the statistical pattern of Fig. 2. Such cases are indeed all the more improbable given that $\mathrm{n}$ is large, but they can still occur. Therefore just witnessing a fixation in one particular large population does not warrant us against such a case. 
Regarding our two kinds of questions distinguished before [namely, either about outcomes of evolutionary models in population genetics (2) or about investigation of an extant population of organisms (1)], this case can clearly pertain to the former. It would then be a case where the less fit allele goes to fixation, that is, unmistakably a case of drift. However, if the case pertains to the latter (1), i.e. an investigation of an extant population of organisms in its ecological context, one can still wonder whether it is indeed an event of drift, for the following reason.

Suppose we observe an allele or a trait fixed in a population and enquire ecologically about the robust causes of life and death, i.e. resource gathering, predation, competition, mates, etc., and that we indeed find that the highest fitness types (as defined by these selective pressures) did not go to fixation. Fitness here is computed by considering the chances reliable factors have to impinge on different types of traits (trait values, alleles, etc.). But the way chance is interpreted here will make a difference to the status of such a case.

(a) If one holds a subjective interpretation of probability, then these subjective chances measure how much we are entitled to believe that they will indeed have such a specific effect. However, (b) if chances are objective, then what is involved in the fitnesses are the objective probabilities that such selective pressures can have such average effect (in the following I suppose we hold here a propensity interpretation).

In this second view (b), there is a possibility that real propensities of ecological factors are not the ones we have hypothesized. So it may be that this case is indeed an example of selection: yet we forgot to include some selective pressures, and our estimation of the chances were wrong. But in the first view (a), the probabilities we ascribed to various outcomes of life experiences for various types in their ecological environments are our degrees of beliefs in these outcomes and therefore they can not be different from what we judge (assuming we judge rationally).

Hence with the objective interpretation (b), this problematic case appears as a possible case of selection (because we may be wrong about the selective pressures and then on the fitnesses); whereas with the subjective interpretation of probabilities (a) this case appears as a case of random genetic drift. For the moment nothing from evolutionary biology compels us to chose between these rival interpretations.

So even if the population is large there is still some opacity of selection and drift. But inversely this could be a reason for deciding for a subjective interpretation of probabilities because it decreases the opacity, thus allowing one to see such example as a case of drift.

\subsubsection{Problematic Case \#2}

The fittest trait went to fixation, but not because of the individuals facing the reliable factors (e.g. environmental demands) better than the others-so not because of the causes of fitness. Here some people could say that it is because of drift that the result expected by selection is reached, or that drift mimics selection. Just observing a large population where a trait gets fixed may not warrant us against such a case of 
drift mimicking selection as long as we do not know the detailed ecology of the process.

This problematic case only concerns the second kind of question i.e. investigating evolution in a population of extant organisms since the causes of fitness are exogenous to population genetics. It also differs from the previous problematic case \#1 because it is not at first sight obvious to classify it. Regarding our sequence of populations in the sample according to the signature of selection (in Sect. 3 above), it is a case of " $\mathrm{X}$ is here by selection" (since the fittest actually goes to fixation). Yet it seems that there is a discrepancy between the actual ecological details and the result of the process in terms of frequencies. Millstein here would say that since the selective pressures were not responsible of the highest frequency of trait $\mathrm{X}$, on the side of the process (vs. the product), this is not a case of selection.

However, as in the previous case, there is an interpretation that confirms this case as one of natural selection, and an interpretation that bears less obvious consequences. A first interpretation argues that we may have not identified all of the relevant selective pressures, but that they are actually redundant with the ones we know (and the basis upon which we initially computed fitness). For example, the tallest did not get more food than others, so their competitive ability (with regard to gaining more food) did not cause the increase in frequency of this trait value within this specific population. But we have overlooked (or did not know) that increased height attracted more mates on a regular basis. Therefore being there because of this reliable cause means that selection for tallness has been acting (but for a different ecological reason from the one originally thought). It is still a case of selection.

The other interpretation states that there were, in fact, many random events that caused differential rates of birth and death for the various types that are exactly the same as the rates that would have otherwise been caused by the more reliable factors (i.e. the selective pressures), and these chance events had no specific reason to impinge in such a way on the differential reproductive rates (e.g., were we to re-run the evolutionary process, the result would be very different). According to the DM account this is clearly a case of drift ( $\mathrm{X}$ is not here because of what it is or is doing biologically). Of course, by the law of large numbers such a case is very improbable, but there is still a possibility that it does happen.

\subsection{Two Perspectives on These Issues}

\subsubsection{Defining the Problem}

These two problematic cases are related to the way we understand probabilities in evolutionary theory. By using the concept of "expected fitnesses" they could indeed be formulated in a shorter manner which emphasizes their difference. Thus in the first problematic case (\#1) the realized fitnesses are not the expected fitnesses (see Burian 1983 for a detailed discussion of expected fitness and its subtypes). That is why changing our conception of probabilities, hence of the word "expected", changes the status of this case. In the second problematic case (\#2), the realized fitnesses are the expected fitnesses, but not because of the reasons why the expected fitnesses are what they are. Here interpreting the probabilities may therefore be less 
consequential since the relationship between "expected" and "realized" is not at stake.

These cases are also different because they concern two aspects of selection (as I have described it in the first section). I argued that the causes of selection cause fitness values, which in turn predict frequency changes. The problem with seeing a given case of fixation in a large population as an instance of selection can arise either at the level of the relation between fitness values and actual offspring number and then frequency change, which means realized fitness does not match expected fitness as in case \#1; or at the level of the relation between fitness values and causes of selection, expected and realized fitnesses being identical as in case \#2.

Hence, given these problematic cases is it justified to say that large populations allow us to overcome the epistemic opacity of selection and drift, and to clearly state that a trait is here because of natural selection? In other terms, if we do have a large population where a trait went to fixation how rational is it to dismiss the hypothesis that we are observing either problematic case \#1 or problematic case \#2?

\subsubsection{Summing Up: Defenses of the Inference from Large Populations}

The previous discussion concluded that provided we can investigate some ecological context, if the realized fitness are not the expected fitnesses then, according to our view of probability, we can either confidently say that it is a case of drift or leave it undecided if we hold an objective view of probabilities. To deal with cases where we can't know an ecological context, or where indeed the fittest go to fixation, we need a further argument, which is the following.

Suppose that we have a very long series of (replicated) large sized populations $P_{i}$ $(1 \ldots \mathrm{i} \ldots \mathrm{k})$ of the size of our actual population, $\mathrm{n}$. In some of these populations $\left(\mathrm{P}_{\mathrm{j}}\right)$ the expected fitnesses ${ }^{16}$ of traits are not reflected by the frequencies, meaning that the reliable causal factors don't have their expected effects on traits frequencies, but the weak law of large numbers states that if $n$ is large enough the chances that indeed these expected effects occur hence the expected fitnesses were realized are very high. Hence considering now the frequencies of populations in the set of populations, it means that the measure of $\left(\mathrm{P}_{\mathrm{j}}\right)$ is very small. Now the actual population $\mathrm{P}_{\mathrm{o}}$ can be viewed as a population randomly picked from this series $\left(\mathrm{P}_{\mathrm{i}}\right)$. The chances that $\left(\mathrm{P}_{\mathrm{o}}\right)$ is precisely one of these populations $\left(\mathrm{P}_{\mathrm{j}}\right)$ in which the reliable factors did not have their expected average result when aggregated, are very low. So it is rational to believe that the actual population is not one of these populations in which random genetic drift let the least fit go to fixation and produced the observed trait values.

Referring to Fig. 3, imagine a series $\left(\mathrm{P}_{\mathrm{i}}\right)$ of populations of size $\mathrm{n}$ (where $\mathrm{n}$ is a large value). The likelihood of randomly picking from such a set one population that will be such that the least fit trait value went to fixation in this population (since in such a set the measure of this subset is very small) is small, and will, and further decrease in probability with increased value of $n$ (this answers problematic case \#1). Moreover, chances that we are in a population where the reliable factors fail to have

$\overline{16}$ Fitness always means expected fitness, in the following. 
their expected effects, even though the trait frequencies in the end correspond to expected fitnesses, are equally very low (answering problematic case \#2). Therefore it is indeed rational to consider that the actual population is not one of these exceptional cases \#1 or \#2. Thus a way of dealing with epistemic opacity is by considering that we have a very high probability of being correct when inferring selection from fixation in large populations ${ }^{17}$.

Moreover, if now we consider genuine biological traits occurring in real ecological contexts, assuming (plausibly) that a trait is underpinned by several alleles it is easy to compute the probability that all these alleles came to fixation together because of drift and we see that this probability-the product of several numbers very close to 0 - is so low (in a large population) that we can neglect it. The point I am making is not that large populations provide us with a better knowledge of environmental demands, reliable factors, causes of fitness etc. Rather, even if we do not know these reliable factors, we can still be confident that the increase in frequencies of some traits is in fact due to such factors that are constant across possible worlds (even if we have yet to identify them precisely) and not due to other less counterfactually constant factors, and then, is to be ascribed to natural selection. This idea-that in large populations when a trait goes to fixation (assuming it's heritable etc., i.e. all conditions for the existence of natural selection are satisfied) it is extremely probable that this is due to natural selection-is in no way different here from any usual scientific case where we deal with large sets and claim that "it is the case that A because the probability of non-A in such a large set is extremely low". 18

Even if in principle there is an epistemic opacity of selection and drift, large populations give us principled and robust reasons to overcome such opacity; this explains why biologists usually think that they have genuinely established the fact that some trait is present in a population because of selection or because of drift and then can in turn legitimately say that a given trait is an adaptation.

\section{Conclusion}

While the overall conclusion of the statistical interpretation of natural selection (according to which selection is not another process acting upon or within populations) is correct, the consequence that drift and selection are statistical properties whose evaluation can not be done on objective grounds is challengeable on the basis of the conceptual distinction between selection and drift made by the

\footnotetext{
${ }^{17}$ Decomposing the population into subpopulation and checking the action of selection and drift may raise problems, as investigated by Walsh (2007, 2010); the question is addressed in Huneman (2013), and Abrams (2013) indicates also a solution that concurs with consequences of the present paper.

18 Statistical mechanics is full of such kinds of reasoning, that support the principle of entropy: crudely stated we consider the repartitions of states of the sets of molecules that realize a lower entropy as hugely more numerous than others, so that the probability of a change in the direction of increasing entropy is extremely higher than the probability of the opposite. Thus the same standards of rationality that entitle us to say that entropy increases, should entitle us to consider that in large populations fixations of traits are due to natural selection.
} 
DM account assumed here. Inscrutability-in the sense (c) of organisms level indistinguishability - is a metaphysical feature of both natural selection and random genetic drift. In contrast, opacity - that instantiates the indistinguishability in sense (a) \& (b) according to statisticalists-is an epistemological feature that can sometimes be reduced and that accounts for the fact that selection and drift appear as statistical and not causal properties [something that the sense (a) of inscrutability is focused upon]. Even though an individual-based distinction of selection and drift is metaphysically impossible, the in-principle opacity of the distinction between them is an epistemological one. It rests on the fact that the causes of fitness, or "causes of selection" (Wade and Kalisz 1990) are not univocally accessible when biologists deal with an individual population. ${ }^{19}$

\section{References}

Abrams, M. (2013). Populations and pigeons: Prosaic pluralism about evolutionary causes. Studies in History and Philosophy of Biological and Biomedical Sciences, 44(2013), 294-301.

Ariew, A., \& Lewontin, R. C. (2004). The confusions of fitness. British Journal for the Philosophy of Science, 55, 347-363.

Beatty, J. (1994). Chance and Natural Selection. Philosophy of Science, 51, 183-211.

Brandon, R. (1990). Adaptation and environment. Princeton: Princeton University Press.

Bouchard, F., \& Rosenberg, A. (2004). Fitness, probability and the principles of natural selection. British Journal for Philosophy of Science, 55, 693-712.

Burian, R. M. (1983). Adaptation. In M. Grene (Ed.), Dimensions of darwinism (pp. 287-314). New York: Cambridge University Press.

Collins, J., Hall, N., \& Paul, L. A. (Eds.). (2004). Causation and counterfactuals. Cambridge: MIT Press.

Depew, D. (2013). Conceptual change and the rhetoric of evolutionary theory: "Force talk" as a case study and challengefor science pedagogy. In Kampourakis, K. (Ed.), The philosophy of biology: A companion for educators (pp.121-144). Dordrecht:Springer.

Dietrich, M. (2013). Molecular evolution. In Kampourakis, K. (Ed.), The philosophy of biology: A companion for educators (pp. 239-248). Dordrecht: Springer.

Endler, J. (1986). Natural selection in the wild. Princeton: Princeton University Press.

Gillespie, J. (2004). Population genetics. New York: Oxford University Press.

Glennan, S. (2009). Productivity, relevance and natural selection. Biology and Philosophy, 24(3), $325-340$.

Harvey, P. H., \& Pagel, M. D. (1991). The comparative method in evolutionary biology. Oxford: Oxford University Press.

Huneman, P. (2012). Natural selection: A case for the counterfactual approach. Erkenntnis, 76(2), 171-194.

Huneman, P. (2013). Assessing statistical views of natural selection: Room for non-local causation? Studies in History and Philosophy of Biological and Biomedical Sciences, 44, 604-612.

Lande, R., \& Arnold, S. (1983). The measurement of selection on correlated characters. Evolution, 37(6), 1210-1226.

Lenormand, T., Rousset, F., \& Roze, D. (2008). Stochasticity in evolution. Trends in Ecology and Evolution, 24, 3.

Lenski, R., \& Trevisano, M. (1994). Dynamics of adaptation and diversification: A 10,000- generation experiment with bacterial populations. Proceedings of the National Academy of Sciences of the United States of America, 91, 6808-6814.

Lewens, T. (2010). The natures of selection. British Journal for Philosophy of Science, 61(2), 313-333.

19 The author warmly thanks Joseph Berkovitz, Isabelle Drouet and two reviewers for their insightful criticisms, as well as Marshall Abrams, Denis Walsh, Andre Ariew and Grant Ramsey for substantial discussions on these claims and Mark Pexton for a thorough language checking. 
Lewis, D. (1973). Causation. Philosophical papers II. New-York: Oxford University Press.

Lewontin, R., \& Dunn, L. (1960). The evolutionary dynamics of a polymorphism in the house mouse. Genetics, 45, 65-72.

Matthen, M. (2009). Drift and 'statistically abstractive explanations'. Philosophy of Science, 76, 464-487.

Matthen, M., \& Ariew, A. (2002). Two ways of thinking about natural selection. Journal of Philosophy, 49(2), 55-83.

Millstein, R. (2002). Are random drift and natural selection conceptually distinct? Biology and Philosophy, 17(1), 33-53.

Millstein, R. (2006). Natural selection as a population-level causal process. British Journal for the Philosophy of Science, 57, 627-653.

Millstein, R., Skipper, R., \& Dietrich, M. (2009). (Mis)interpreting mathematical models: Drift as a physical process. Philosophy and Theory in Biology, 1. doi:10.3998/ptb.6959004.0001.002.

Orr, H. A. (2009). Fitness and its role in evolutionary genetics. Nature Reviews Genetics, 10, 531-539.

Plutynski, A. (2007). Drift: A historical and conceptual overview. Biological Theory, 2(2), 156-167.

Ramsey, G. (2013). Organisms, traits, and population subdivisions: Two arguments against the causal conception of fitness? British Journal for the Philosophy of Science, 54, 589-608.

Riesman, K., \& Forber, P. (2005). Manipulation and the causes of evolution. Philosophy of Science, 72, 1113-1123.

Rosenberg, A. (1994). Instrumental biology, or the disunity of science. Chicago: University of Chicago Press.

Shapiro, L., \& Sober, E. (2007). Epiphenomenalism-The Do's and the Don'ts. In G. Wolters \& P. Machamer (Eds.), Studies in causality: Historical and contemporary. Pittsburgh: University of Pittsburgh Press.

Sober, E. (1984). The nature of selection. Cambridge: MIT Press.

Stephens, C. (2004). Selection, drift and the "forces" of evolution. Philosophy of Science, 71, 550-570.

Wade, M., \& Kalisz, S. (1990). The causes of natural selection. Evolution, 44(8), 1947-1955.

Walsh, D. (2007). The pomp of superfluous causes: The interpretation of evolutionary theory. Philosophy of Science, 74, 281-303.

Walsh, D. M. (2010). Not a sure thing: Fitness, probability, and causation. Philosophy of Science, 77(2), 141-171.

Walsh, D. (forthcoming). Variance explanations. Erkenntnis, doi:10.1007/s10670-014-9680-3.

Walsh, D., Lewens, T., \& Ariew, A. (2002). Trials of life: Natural selection and random drift. Philosophy of Science, 69, 452-473.

Woodward, J. (1999). Causal interpretation in systems of equations. Synthese, 121, 199-247.

Woodward, J. (2003). Making things happen. New-York: Oxford University Press. 